\title{
Desfolha em videiras americanas e viníferas na fase de pré-maturação dos frutos
}

\author{
Defoliation of american and vinifera grapevines in the pre ripening of fruits
}

\author{
Rafael Anzanello $^{\mathrm{I}^{*}}$ Paulo Vitor Dutra de Souza ${ }^{\mathrm{I}}$ Pedro Ferreira Coelho $^{\mathrm{I}}$
}

- NOTA -

\section{RESUMO}

Foram avaliadas possiveis interferências que a localização da folha removida mediante desfolha em videira apresenta sobre o rendimento e qualidade dos frutos. As cultivares 'Niagara Branca'e 'Concord', ambas Vitis labrusca, $e$ 'Cabernet Sauvignon'e 'Merlot', ambas Vitis vinifera, foram utilizadas em sistema de condução em espaldeira, na safra 2006/2007. O experimento foi conduzido em delineamento de blocos casualizados, com quatro repetições e quatro plantas por parcela, sendo testados 4 tratamentos: $T 1$ (testemunha sem desfolha), $T 2$ (retirada das folhas localizadas opostas aos cachos), T3 (retirada das folhas do sarmento localizadas abaixo dos cachos) e T4 (retirada das folhas do sarmento localizadas abaixo e opostas aos cachos). As cultivares da espécie Vitis labrusca receberam um tratamento adicional T5, constando na retirada das folhas acima dos cachos. A aplicação dos tratamentos foi realizada na fase de mudança de cor das bagas para as cultivares 'Concord', 'Merlot' e 'Cabernet Sauvignon' e início do amolecimento das bagas para a cultivar 'Niagara Branca'. Avaliaram-se, após a colheita, a produção por planta, a massa dos cachos, os teores de sólidos solúveis (SS), a acidez. titulável, (AT) e o pH dos frutos. Observou-se que a desfolha até a altura do cacho não influenciou na quantidade e na qualidade dos frutos. Porém, quando a desfolha foi realizada acima dos cachos, ocorreu um atraso na maturação das uvas americanas. Essa condição implica não ser necessária a desfolha seletiva até a altura dos cachos nas videiras estudadas, quando realizada na fase início de amadurecimento dos frutos.

Palavras-chave: poda verde, Vitis sp., maturação, fisiologia vegetal.

\begin{abstract}
This study aimed to evaluate the interference that the location of the leaf removed by defolation in grapevine has on fruits quality and yield. Such experiment was performed using Niagara Branca and Concord, both Vitis labrusca, and Cabernet Sauvignon e Merlot, both Vitis vinifera, trained in a unilateral cordon system during the 2006/2007 harvesting. The experiment was disposed in a randomized block design, with four replications and four plants per plot. The following treatments were tested: $T 1$ (no defoliation), T2 (removal of leaves located in the opposite side of the grapes), T3 (removal of leaves located under the grapes) and T4 (removal of leaves located under and in the opposite side of the grapes). The Vitis labrusca received an additional treatment (T5) consisting of leaves removed above the grapes. These treatments were applied during the change of berries color for Concord, Cabernet Sauvignon and Merlot grapes and in the beginning of berries softening for Niagara Branca grape. After the harvesting, the grapevine production, cluster weight, total soluble solids, total titratable acidity and $\mathrm{pH}$ were evaluated. It was observed that the defoliation until the grapes height did not influence the quantity and quality of grapes. However, when the defoliation was carried out above the clusters, there was a delay in the maturation of american grapes. This condition shows that is not necessary a selective desfolation until the clusters height on grapevines, when it is realized during the initial fruit ripening phase.
\end{abstract}

Key words: green pruning, Vitis sp., maturation, plant physiology.

A poda verde é realizada em videiras com o objetivo de equilibrar o desenvolvimento vegetativo e

IDepartamento de Horticultura e Silvicultura, Faculdade de Agronomia, Universidade Federal do Rio Grande do Sul (UFRGS), Av. Bento Gonçalves, 7712, Bairro Agronomia, CP 15100, 91501-970, Porto Alegre, RS, Brasil. E-mail: ranzanello@yahoo.com.br. *Autor para correspondência. 
a produção, visando à melhoria da qualidade da uva. Tal operação é feita com diversas finalidades e de vários modos. Emprega-se para complementar a poda seca durante a formação da planta, para facilitar a penetração de luz, de ar e de calor, para garantir a fecundação das flores, para diminuir a incidência de moléstias e para economizar fungicidas. A desbrota, a desponta e a desfolha são modalidades de poda verde que interferem nas características do dossel vegetativo (GIOVANINNI, 2008).

$\mathrm{Na}$ desbrota, são eliminados os brotos que surgem das gemas dormentes do tronco e dos braços velhos e os brotos improdutivos, enfraquecidos. Ela é realizada para proporcionar melhor aproveitamento das substâncias de reserva da videira e facilitar a realização da poda de inverno do ano seguinte, o que melhora a distribuição e o desenvolvimento dos ramos selecionados (PONI, 2003). O desponte baseia-se na supressão das extremidades dos ramos para diminuir a dominância apical, favorecer a maturação das gemas basais, aumentar a massa média dos cachos e a qualidade da uva (PONI et al., 2005). Já, a desfolha consta na eliminação de folhas, principalmente as situadas próximas aos cachos, para proporcionar arejamento e insolação na região dos frutos, promovendo melhores condições para a sua maturação (GIOVANINNI, 2008).

De acordo com a posição em que se encontram as folhas a serem removidas do ramo durante a desfolha, alterações nutricionais e metabólicas nos frutos podem ocorrer (BAVARESCO et al., 2008).

Em videiras, a retirada indiscriminada de folhas na região dos cachos é uma prática usual entre os produtores (MANDELLI et al., 2008). Porém, o arranjo das folhas remanescentes quando da prática da desfolha, associada às relações produtivas da planta, ainda encontram-se desconhecidas. Nesse contexto, o presente trabalho visou a avaliar as possíveis interferências que a localização da folha removida mediante desfolha na cultura da videira apresenta sobre o rendimento e a qualidade dos frutos.

Realizaram-se dois experimentos. Em um deles, utilizaram-se duas cultivares de uvas americanas (Vitis labrusca), 'Niagara Branca' e 'Concord', enxertadas sobre o porta-enxerto 101-14mgt, conduzidas em espaldeira simples no espaçamento de 1,20 x 3,00m, em vinhedo localizado em Eldorado do Sul - RS. Em outro experimento, testaram-se duas cultivares de uvas viníferas (Vitis vinifera), 'Cabernet Sauvignon' e 'Merlot', enxertadas sobre o porta-enxerto 1103 Paulsen, conduzidas em espaldeira simples no espaçamento de $1,50 \times 3,50 \mathrm{~m}$, em vinhedo localizado na região da Serra Gaúcha, município de Veranópolis - RS.
Os experimentos foram conduzidos na safra 2006/2007, em delineamento de blocos casualizados com quatro repetições e quatro plantas por parcela, testando-se quatro tratamentos: T1 (testemunha - sem desfolha); T2 (retirada das folhas opostas aos cachos - remoção de aproximadamente 70 folhas/planta); T3 (retirada das folhas do sarmento localizadas abaixo dos cachos - remoção de aproximadamente 50 folhas/planta) e T4 (retirada das folhas do sarmento localizadas abaixo e opostas aos cachos - remoção de aproximadamente 120 folhas/planta). As Vitis labrusca receberam um tratamento adicional (T5), constando da retirada de todas das folhas localizadas acima dos cachos, mantendo-se as demais. Neste tratamento, foram removidas aproximadamente 240 folhas/planta. Os tratamentos foram aplicados na fase de mudança de cor das bagas para a 'Concord', 'Cabernet Sauvignon' e 'Merlot' e na fase de amolecimento das bagas para a 'Niagara Branca'.

Avaliaram-se, após a colheita, a produção por planta (kg); a massa média dos cachos (g); os teores de sólidos solúveis (SS); a acidez titulável (AT) e; o pH dos frutos.

A produção por planta foi obtida pela pesagem dos cachos colhidos em balança eletrônica. A massa média dos cachos foi obtida pela divisão entre a produção por planta e o número de cachos por planta. Para a análise da composição química dos frutos, foram coletados dez cachos de uva por unidade experimental. $\mathrm{O}$ valor de SS foi determinado em refratômetro de mesa, a partir de amostra de 20 microlitros de mosto. AAT foi determinada por titulação com $\mathrm{NaOH} 0,1 \mathrm{~N}$, conforme CATALUÑA (1984). As variáveis de produção quantitativa e qualitativa foram submetidas à análise da variância. Os resultados com diferenças significativas, pelo teste " $F$ ", tiveram suas médias submetidas ao teste de Tukey ao nível de 5\% de probabilidade.

De acordo com os resultados, a cultivar 'Niagara Branca' apresentou maior massa de cacho e maior produção por planta comparativamente à 'Concord' (Tabela 1). As variáveis qualitativas (SS e AT) foram semelhantes entre as duas cultivares (Tabela 1). Em relação às uvas viníferas, a 'Merlot' apresentou menor AT se comparada à 'Cabernet Sauvignon', não havendo diferenças para os teores de sólidos solúveis, pH e variáveis quantitativas (Tabela 1). As diferenças apontadas na produção por planta e massa por cacho, bem como na evolução da maturação entre as cultivares estudadas, provavelmente deve-se às características genéticas inerentes às cultivares (CAMARGO \& DIAS, 1984). 
Tabela 1 - Variáveis de produção quantitativa e qualitativa das cultivares 'Niagara Branca' (NB), 'Concord' (C), 'Cabernet Sauvignon’ (CS) e 'Merlot' (M) submetidas a diferentes tratamentos de desfolha. Eldorado do Sul e Veranópolis, 2007.

\begin{tabular}{|c|c|c|c|c|c|c|c|c|c|c|}
\hline \multirow{3}{*}{ Trat } & \multicolumn{4}{|c|}{--------------Variáveis quantitativas-------------- } & \multicolumn{6}{|c|}{ 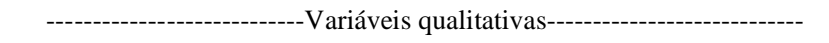 } \\
\hline & \multicolumn{2}{|c|}{ Produção (kg planta $\left.{ }^{-1}\right)$} & \multicolumn{2}{|c|}{ Massa $\left(\mathrm{g} \mathrm{cacho}^{-1}\right)$} & \multicolumn{2}{|c|}{ Acidez total $\left(\right.$ meq $\left.\mathrm{L}^{-1}\right)$} & \multicolumn{2}{|c|}{ Sólidos solúveis $\left({ }^{\circ} \mathrm{Brix}\right)$} & \multicolumn{2}{|c|}{$\mathrm{pH}$} \\
\hline & NB & $\mathrm{C}$ & NB & $\mathrm{C}$ & NB & $\mathrm{C}$ & NB & $\mathrm{C}$ & NB & $\mathrm{C}$ \\
\hline $\mathrm{T} 1^{1}$ & $10,37 \mathrm{aA}^{2}$ & $8,06 \mathrm{aB}$ & $132,88 \mathrm{aA}$ & $85,15 \mathrm{aB}$ & $81,1 \mathrm{bA}$ & $83,6 \mathrm{bA}$ & $15,83 \mathrm{aA}$ & $14,91 \mathrm{aA}$ & $3,12 \mathrm{aA}$ & $3,10 \mathrm{aA}$ \\
\hline $\mathrm{T} 2$ & $10,82 \mathrm{aA}$ & $8,42 \mathrm{aB}$ & $135,25 \mathrm{aA}$ & $90,10 \mathrm{aB}$ & $77,3 \mathrm{bA}$ & $81,6 \mathrm{bA}$ & $15,45 \mathrm{aA}$ & $14,55 \mathrm{aA}$ & $3,12 \mathrm{aA}$ & $3,02 \mathrm{aA}$ \\
\hline $\mathrm{T} 3$ & $11,01 \mathrm{aA}$ & $8,94 \mathrm{aB}$ & $141,34 \mathrm{aA}$ & $87,23 \mathrm{aB}$ & $79,1 \mathrm{bA}$ & $84,6 \mathrm{bA}$ & $15,41 \mathrm{aA}$ & $14,95 \mathrm{aA}$ & 3,09 aA & $3,05 \mathrm{aA}$ \\
\hline $\mathrm{T} 4$ & $10,96 \mathrm{aA}$ & $8,36 \mathrm{aB}$ & $129,43 \mathrm{aA}$ & $91,13 \mathrm{aB}$ & $78,6 \mathrm{bA}$ & $82,2 \mathrm{bA}$ & $15,83 \mathrm{aA}$ & $14,89 \mathrm{aA}$ & $3,14 \mathrm{aA}$ & $3,04 \mathrm{aA}$ \\
\hline T5 & $9,22 \mathrm{bA}$ & $7,10 \mathrm{bB}$ & $108,54 \mathrm{bA}$ & $74,10 \mathrm{bB}$ & $100,5 \mathrm{aA}$ & $109,9 \mathrm{aA}$ & $11,58 \mathrm{bA}$ & $11,89 \mathrm{bA}$ & $2,95 \mathrm{bA}$ & $2,91 \mathrm{bA}$ \\
\hline \multirow[t]{2}{*}{$\mathrm{CV}(\%)$} & \multicolumn{2}{|c|}{21,45} & \multicolumn{2}{|c|}{18,87} & \multicolumn{2}{|c|}{13,84} & \multicolumn{2}{|c|}{15,57} & \multicolumn{2}{|c|}{14,63} \\
\hline & $\mathrm{M}$ & CS & M & CS & $\mathrm{M}$ & CS & M & CS & $\mathrm{M}$ & CS \\
\hline $\mathrm{T} 1$ & $8,52 \mathrm{aA}$ & $7,15 \mathrm{aA}$ & $130,15 \mathrm{aA}$ & $124,23 \mathrm{aA}$ & 95,6 aA & $115,3 \mathrm{aB}$ & $19,66 \mathrm{aA}$ & $19,33 \mathrm{aA}$ & $3,41 \mathrm{aA}$ & $3,40 \mathrm{aA}$ \\
\hline $\mathrm{T} 2$ & $8,79 \mathrm{aA}$ & 8,66 aA & $138,77 \mathrm{aA}$ & $120,43 \mathrm{aA}$ & 94,8 aA & $117,3 \mathrm{aB}$ & $19,79 \mathrm{aA}$ & $18,62 \mathrm{aA}$ & $3,43 \mathrm{aA}$ & $3,36 \mathrm{aA}$ \\
\hline $\mathrm{T} 3$ & $9,01 \mathrm{aA}$ & $8,22 \mathrm{aA}$ & $129,32 \mathrm{aA}$ & $118,98 \mathrm{aA}$ & $99,0 \mathrm{aA}$ & $115,8 \mathrm{aB}$ & $19,70 \mathrm{aA}$ & $18,83 \mathrm{aA}$ & $3,40 \mathrm{aA}$ & $3,33 \mathrm{aA}$ \\
\hline $\mathrm{T} 4$ & $9,34 \mathrm{aA}$ & 7,89 aA & $141,12 \mathrm{aA}$ & $127,37 \mathrm{aA}$ & $95,0 \mathrm{aA}$ & $113,1 \mathrm{aB}$ & $19,41 \mathrm{aA}$ & $18,70 \mathrm{aA}$ & $3,14 \mathrm{AA}$ & $3,36 \mathrm{aA}$ \\
\hline $\mathrm{CV}(\%)$ & \multicolumn{2}{|c|}{28,17} & \multicolumn{2}{|c|}{24,43} & \multicolumn{2}{|c|}{11,81} & \multicolumn{2}{|c|}{13,73} & \multicolumn{2}{|c|}{12,05} \\
\hline
\end{tabular}

(') T1 (testemunha - sem desfolha), T2 (retirada das folhas apostas aos cachos), T3 (retirada das folhas do sarmento localizadas abaixo dos cachos) e T4 (retirada das folhas do sarmento localizadas abaixo e opostas aos cachos), T5 (retirada das folhas acima dos cachos). $\left({ }^{2}\right)$ Médias, dentro de cada variável, seguidas de mesma letra, minúsculas na coluna e maiúsculas na linha, não diferem entre si pelo teste Tukey em nível de $5 \%$ de probabilidade.

Quanto aos tratamentos de desfolha, independentemente da cultivar de Vitis labrusca, apenas o tratamento 5 (desfolha acima da altura dos cachos) diferiu dos demais, ocasionando menor produção por planta e menor massa por cacho (Tabela 1). Além disso, o tratamento T5 conferiu aos frutos menor teor de $\mathrm{SS}$ associado à maior $\mathrm{AT}$ e menor $\mathrm{pH}$ do mosto (Tabela 1). Isso pode ser explicado pela severa redução da área fotossintética provocada nos ramos das plantas submetidas ao tratamento T5, associado à alteração temporária da rota dos fotoassimilados, os quais em ramos com desfolha até a altura do cacho foram translocados preferencialmente aos frutos, enquanto que nos desfolhados acima dos cachos devem ter sido desviados para promover brotações das gemas apicais dos ramos, para aportar material fotossintético para a nutrição dos cachos em desenvolvimento.

Os tratamentos de desfolha realizados até a altura dos cachos nas cultivares viníferas não influenciaram na produção por planta e na massa dos cachos, nem a AT, SS e pH do mosto (Tabela 1). Pesquisas que abordam o tema poda verde da videira, ressaltam a importância da época e intensidade de sua realização e, em função delas, podem-se obter resultados distintos nas características físico-químicas dos frutos (MAIN \& MORRIS, 2004; PONI et al., 2005; BAVARESCO et al., 2008). Tais evidências não foram observadas neste estudo, o que pode estar associado a não avaliação de diferentes épocas, mas sim somente diferentes intensidades de desfolha.

De modo geral, a desfolha até a altura do cacho não influenciou na produção para os diferentes grupos de cultivares de videira (Tabela 1), o que indica que a desfolha pode ser um determinante para a coloração das bagas e para aumentar o arejamento próximo aos cachos, mas não interfere nas características internas das bagas, quando ela é realizada na fase de mudança de cor e/ou amolecimento dos frutos. Essa condição implica não ser necessária uma desfolha seletiva na cultura da videira, tornando a prática mais rápida e menos dispendiosa, quando feita no início da maturação dos cachos.

Com este trabalho, conclui-se que a remoção localizada das folhas até a altura do cacho, no início do amadurecimento das bagas, não altera as variáveis quantitativas e qualitativas dos frutos. Além disso, verifica-se que, para as cultivares 'Niagara Branca' e 'Concord', a desfolha realizada acima dos cachos provoca um atraso na maturação das uvas e afeta negativamente as características físico-químicas dos frutos.

\section{AGRADECIMENTOS}

Ao Conselho Nacional de Desenvolvimento Científico e Tecnológico (CNPq). 


\section{REFERÊNCIAS}

BAVARESCO, L. et al. Effect of leaf removal on grape yield, berry composition, and stilbene concentration. American Journal of Enology and Viticulture, v.59, n.3, p.292-298, 2008. Disponível em: <http://www.ajevonline.org/cgi/reprint/ 59/3/292>. Acesso em: 27 ago. 2010

CATALUÑA, E. Uvas e vinhos. Rio de Janeiro: Globo, 1984. $230 \mathrm{p}$.

CAMARGO, U.A.; DIAS, M.F. Identificação varietal de algumas videiras cultivadas no Rio Grande do Sul. Bento Gonçalves: CNPUV- Embrapa, 1984. 47p.

GIOVANINNI, E. Produção de uvas para vinhos, suco e mesa. 3.ed. Porto Alegre: Renascença, 2008. 364p.

MAIN, G.L.; MORRIS, J.R. Leaf-removal effects on Cynthiana yield, juice composition, and wine composition. American
Journal of Enology and Viticulture, v.55, n.2, p.147-152, 2004. Disponível em: <http://www.ajevonline.org/cgi/reprint/ 55/2/147>. Acesso em: 5 set. 2010.

MANDELLI, F. et al. Efeito da poda verde na composição físico-química do mosto da uva Merlot. Revista Brasileira de Fruticultura, v.30, p.667-674, 2008. Disponível em: < ht t p://www.scielo.br/scielo.php? pid=S 0100 $29452008000300018 \&$ script=sci_arttext $>$.Acesso em: 19 set. 2010. doi: 10.1590/S0100-29452008000300018.

PONI, S. La potatura verde nel vigneto: aspetti fisiologici e colturali. L'Informatore Agrario, v.59, n.26, p.37-49, 2003.

PONI, S. et al. Effects of early removal on cluster morphology, shoot efficiency and grape quality in two Vitis vinifera cultivars. Acta Horticulturae, n.689, p.217-226, 2005. Disponível em: <http://www.actahort.org/members/showpdf? session=20373>. Acesso em: 31 jul. 2010. 